\title{
Research on Applications of Virtual Reality Technology in Medical
}

\section{Education}

\author{
Zhuo Zhang ${ }^{1, a}, \mathrm{Ge} \mathrm{Gao}^{1, \mathrm{~b}}$, Haiyan Zhang ${ }^{1, \mathrm{c}}$ \\ ${ }^{1}$ Urology Department, China Japan Union Hospital of Jilin University, Changchun City, Jilin \\ Province, China, 130033 \\ ${ }^{2}$ Pathology Department, China Japan Union Hospital of Jilin University, Changchun City, Jilin \\ Province, China, 130033 \\ ${ }^{3}$ Gastrointestinal Department, China Japan Union Hospital of Jilin University, Changchun City, \\ Jilin Province, China, 130033 \\ Corresponding Author: ZHANG Haiyan,3455064@qq.com
}

Keywords: Virtual Reality Technology, Medical Education, Medical Virtual Teaching

\begin{abstract}
Virtual reality is a computer system which can create and experience the virtual world. People can operate, control and reach some special purposes in this system. This paper firstly introduces the concept and advantages of the virtual reality technology, and then explores the applications of virtual reality in medical instruction, including virtual observation instruction, virtual experiment instruction, virtual anatomy instruction and virtual operation instruction, to provide some references for the related researchers.
\end{abstract}

\section{Concept of Virtual Reality Technology}

Virtual reality is an advanced computer human interface, which is characterized by immersion, interaction and imagination. It integrates computer graphics, simulation technology, multimedia technology, artificial intelligence technology, computer network technology, parallel processing technology and sensor technology, visual, auditory, tactile and other sensory function simulation, can make people immersed in a computer generated virtual realm, and to carry out real-time interaction with the language, the gesture of natural way, create a multi-dimensional information space of a humanoid. Users can not only feel in the physical world objectively experienced fidelity through virtual reality system, and can break through space, time and other objective constraints, feel the real world cannot be experienced experience. We can simply understand the virtual reality system: using computer generated human computer interaction system that can give people a variety of sensory stimulation. It includes two aspects: the first is the computer generated virtual environment must be able to provide a variety of sensory stimulation of the senses, can let a person have the feeling of immersion, virtual reality at this stage usually consists of visual, auditory and tactile; the other is the virtual reality system is an advanced interactive system the human-computer interaction is the core of virtual reality.

\section{Advantages of Virtual Reality Technology in Medical Instruction}

Virtual reality technology is one of the core content of educational technology, the fundamental difference with other teaching methods is the virtual reality technology has broken the traditional teacher dominated in the learning process, learners can maximize their initiative and enthusiasm, provide rich resources and convenient space for training of divergent thinking they will learn, 
practice, forming a vivid, lively, active way of teaching, which is any of the traditional teaching way and method of reach, so it has irreplaceable function and role. In Medical University, the traditional teaching method in classroom teaching, the teacher oral communication, on the blackboard presentation, then combined with the teaching model, gestures, pictures, samples and human auxiliary teaching tool, animal experiment, and use the high cost of the autopsy learners to deepen the understanding of the teaching contents, improve their practical ability.

In recent years, the multimedia teaching to the text, it A new force suddenly rises., sound, image, video and animation and other information collection in a body, with innovative teaching methods, lively and vivid teaching form, has become the mainstream of modern teaching methods. With the rapid development of modern science and technology, virtual reality technology will become the next generation of medical colleges and universities in the teaching of the main auxiliary teaching means. The application of virtual reality technology in medical teaching, not only the text, graphics, images, sound and animation together organically, and all-round, multi angle is presented to the learner, continue to stimulate the learner's senses, make them into a new world. In teaching, the problem of teaching method, heuristic method of teaching to fully mobilize the learner's thinking ability, so that their brain in an excited state, to stimulate interest in learning, expand the space of thinking. Under this kind of stimulation, the learner's thinking is activated, clear and smooth, which has laid a good foundation for the cultivation of innovative thinking. The virtual scene virtual reality technology in the discovery teaching can make learners into the problems of environment have, with inspiration to construct virtual situation to guide them to explore, provides the path of discovery learning thinking. Virtual reality teaching not only provides a good human-computer interaction to learners, but also allows learners in the learning process of errors, to correct the error. By mistake to let the learners understand the causes and consequences of the error, and then find a way to solve the error. This process of correcting the error of the training of the learner's analysis, synthesis, induction and other thinking skills, the formation of discovery and heuristic learning style, cultivate a high level of thinking skills. Virtual reality technology can construct the best teaching environment, to learn the abstract and reality together, and tends to display a variety of real learning situations, thus inducing learners instant thinking, stimulate their thinking. In the virtual scene of the virtual reality technology, it illustrates the function of learners in an excited state.

Students can communicate directly with the virtual environment, so as to enrich perceptual knowledge, deepen the understanding of teaching content, thus greatly stimulate students' learning interest and enthusiasm, improve their level of perception. For example, in the study of acupoints positioning, traditional teaching is usually presented to the learner to human physical model of static picture, plastic or rubber, the human body simulation system but the use of virtual reality technology can be generated, multi angle, all-round display of positioning and manipulation of the acupuncture points corresponding to the disease, the needle so, very intuitive, vivid, greatly improving the students' learning enthusiasm.

\section{Applications of Virtual Reality Technology in Medical Education}

Virtual Observation Instruction. The concept of imaging structure of human body structure or digital human body is put forward in the medical field. The construction process of the digital human body, using 3D or higher dimensional medical imaging of human tissue events are stored as a 3D database, and then use computer technology to construct the human body or organization into a three-dimensional image. In this way, we can make a retrospective observation and analysis of the human body and the human body in the digital human body, and this operation can be carried out by modern computer network system. To construct a mechanism of human brain for example, using 
brain magnetic images, the use of virtual reality modeling language for reconstruction of human brain structure, spatial shape, spatial location of brain structures of interest have a more intuitive understanding, and can interact with the interactive operation, to observe the from all directions and angles. The traditional experiment teaching by teachers or drawing charts to explain the experimental principle, the teacher on the blackboard to draw some simple graphs, both time-consuming and lack of authenticity. With the updating of knowledge, it is difficult to keep up with updated charts. At the same time, simple graphs and charts is not big enough, sitting in the back of the classroom for students to see, more to record. R V should be used in teaching, so that some abstract, difficult to understand the theory, the concept of image, specific. As in the structure and function on immune globulin content, the primary structure of our first graphic immune globulin, have some of the characteristics of the students in this structure, continue animation immunoglobulin two level and three level structure, so that students in the mind set up the three-dimensional conformation of immunoglobulin. On this basis, further animation to demonstrate and explain how the immunoglobulin binding antigen, how to combine the organization of the cell.

Virtual Experiment Instruction. Virtual reality technology to make up for the deficiency of the existing teaching conditions, improve the teaching level. Virtual reality technology provides no loss, can use unlimited experimental environment to a great extent to solve the majority of medical occupation college experiment teaching experiment teaching resources of high cost and expensive and easy to damage the problem, it is difficult to reuse, greatly saves the laboratory resources, ease the contradiction between teaching resource and medical skills the train asked, it has an important significance to improve the teaching level of medical occupation education. Medical vocational education is a kind of education which is based on medical skill training, and the training of medical experimental skills is the core of the teaching. Among them, the training of medical experimental skills can be divided into four stages: the orientation, the imitation, the integration and the proficiency of the skill operation. Skill orientation is to set up the orientation image process of each operation link in the mind. With this kind of directional mapping, the learners can be regulated by the image in the actual operation of the subsequent imitation, integration and proficiency stage, and know how to operate. The characteristics of the virtual experimental teaching system can make full use of the idea of virtual reality technology in the design of the process of the experimental skills training were analyzed and illustrated reasonably, which can help students quickly establish a correct orientation image, improve the efficiency of skill training in medical experiments, promote the realization of medical occupation education experimental skill training target.

Virtual Anatomy Instruction. To build a human like computer model of medical science and computer technology combined with the research on digitized virtual human is very significant in digital anatomy, surgery planning and training and many other medical applications. Three dimensional reconstruction and display of the image of continuous anatomical sections is an important method of morphological study. The use of Java for the preparation of three-dimensional reconstruction software based on the network to carry out reconstruction and anatomical virtual. Results the reconstructed images of the skull and facial images are vivid, hierarchical display, can rotate at any angle, and can be repeatedly cut and restored. To realize the reconstruction and virtual anatomy of the skull and facial tissue in the internet network, and provide a new software system for computer aided anatomy. The use of virtual reality technology in teaching can be a lot of teaching information directly reflected the abstract concept of visualization, visualization, save a lot of expensive experimental equipment costs, arouse students' enthusiasm and initiative, brings great flexibility to the teaching design process, can break the limit of time and space, avoid all kinds of 
risk brought by the real experiment or operation, is conducive to the innovation of teaching mode, the development of the teaching environment can be constructed..

Virtual Operation Instruction. The research direction of the virtual surgery as this field is developed, its purpose is to use various kinds of medical image data, using virtual reality technology, the establishment of a simulation environment in the computer, the doctor performed the operation plan, with the help of information in virtual environment walkthrough operation, operation instruction, skills training. Virtual surgery simulator is an important application of virtual reality technology in medicine. Study on the development of virtual surgery simulator fast construction technology of virtual human organ tissue physical model is one of research needs, it can lay the foundation for the development of the research work carried out in the next step of virtual surgery simulator. Taking the simulator of the biological soft tissue as an example, the linear elastic physical model is adopted in the virtual hand. However, the biological soft tissues themselves show the physical characteristics of viscoelasticity. Through the analysis on the biomechanical properties of soft tissue, study the application of viscoelastic model in soft tissue deformation in surgery simulation. Under the premise of minimizing the complexity of the algorithm, the calculation formula of the viscoelastic model is derived, which can be used to simulate the deformation of the soft tissue under the action of external force. At the same time, the fast and accurate collision detection is an important condition to ensure the authenticity of the operation simulation. The real-time and complexity of the operation simulation put forward higher requirements for collision detection. The most widely used method of collision detection is based on the bounding box hierarchy of the fixed direction convex bags. This method not only can effectively improve the speed of collision detection, but also can adapt to the changes of the geometric characteristics and topological structure of soft tissue deformation, and can solve the collision detection problem in the operation simulation.

\section{Conclusion}

The application of virtual reality technology in medical education is an important direction. With the continuous improvement of virtual reality technology and network technology, virtual campus or virtual classroom based on the internet will become a new teaching model in the field of education. The virtual reality technology will be applied more and more widely in the medical education.

\section{References}

[1] Tian Yin, Computer Knowledge and Technology, Vol. 10 (2014) No 32, p.7786-7789

[2] Wang Changzhi, China Education Innovation Herald, Vol. 31 (2012) No 1, p.44-45

[3] Hu Weihong, Liu Daoguang, Wang Qian, Li Meng, Journal of Shandong Youth Administrative Cadres College, Vol. 23 (2007) No 6, p.139-141

[4] Cao Ding, Li Wenjian, Guide of China Medicine, Vol. 11 (2013) No 3, p.367-368 